\title{
EL ACETATO DE MEDROXY PROGESTERONA DE DEPOSITO COMO ANTICONCEPTIVO*
}

\author{
Dr. Alberto Duarte-Contreras, M.D.**
}

\section{I - Introducción}

En 1965 Rice Wray publicó sus primeras observaciones sobre el uso de combinaciones progestágeno-estrógeno en inyección de depósito para el control de la fertilidad: buscaba en esta forma simplificar la anticoncepción hormonal por vía oral conservando la efectividad y mejorando la tolerancia $(10,21)$.

Se emplearon entonces dosis elevadas de Medroxy Progesterona (de la casa Upjohn) sola o con estrógenos en proporciones diferentes que aseguraban la inhibición de la ovulación por períodos de un año, seis meses o tres meses, según se administrasen 1.000, 500 o $150 \mathrm{mg}$. del gestágeno: Su actividad fue óptima pero se observaron amenorreas de duración impredecible.

Por aquellos mismos días aparecían estudios con un preparado gestágeno-estrógeno diferente que se administraba cada 30 días y que evitaba las alteraciones del sangrado mensual: era el acetato-fenido-dihidroxy progesterona (100 mg.) con enantato de estradiol (de 5 a $20 \mathrm{mg}$.) producido por la casa Squibb. Los resultados fueron satisfactorios y los trastornos menstruales mínimos, pero la inyección mensual desanimó a los investigadores (20).
En 1966 Larrañaga presentó sus experiencias con el capronato de hidroxy progesterona o SH 582 de la casa Schering alemana al que atribuyó un efecto anticonceptivo sumamente efectivo (7) a dosis de 200 mg. cada ocho semanas. Pero estudios posteriores demostraron que no ofrecía alta seguridad anticonceptiva (Indice de Pearl 18,6) y que daba marcadas alteraciones sobre los ciclos menstruales; por este motivo se le descontinuó (12).

Se viene utilizando también desde 1966 el enantato de nor etisterona o $\mathrm{SH} 393$ de la casa Schering alemana a la dosis de $200 \mathrm{mg}$. cada 10 semanas; ha resultado de gran efectividad anticonceptiva pero produce también trastornos del ciclo menstrual $(9,13,22)$.

Nosotros iniciamos en 1969 la presente investigación animados por el deseo de escalar un peldaño más en el estudio de los diversos métodos ovulostáticos conocidos (2, 3, 4) empleando el Acetato de Medroxy Progesterona de Depósito (A.M.P.D.) a la

\footnotetext{
* Trabajo presentado ante la Academia Nacional de Medicina para ingresar como Miembro Correspondiente.

* * Médico Jefe del Departamento Gíneco Obstétrico del Hospital San Juan de Dios. Cúcuta, Colombia.
} 
dosis de $150 \mathrm{mg}$. por vía intramuscular cada noventa días.

Sabemos que este preparado de la casa Upjohn es un progestógeno puro, potente, de efecto prolongado $y$ de enérgica inhibición gonadotrópica. Farece que su acción se debe: 1) a la inhibición de la ovulación como resultado de la supresión de gonadotropinas, especialmente de la L.H.; 2 ) a su acción sobre el endometrio, el que se torna en no adecuado para la implantación; y 3) a alteraciones del moco cervical que impiden el paso normal de los espermatozoides.

\section{Material y Métodos}

II. Motivamos 243 pacientes: 148 rechazaron el método alegando una - varias de las siguientes razones: posibilidad de amenorrea, posibilidad de sangrados prolongados, por estar satisfechas con otros métodos, por ser una droga experimental, por posible infertilidad posterior de tiempo no precisado. $Y$ sólamente pudimos estudiar 95 mujeres, todas ellas normales, eumenorreicas, sin trastornos ginecológicos ni endocrinos aparentes: una nulípara y 94 multíparas. Les administramos $150 \mathrm{mg}$. de A.M. P.D. intramuscular cada 90 días.
Antes de iniciar el tratamiento les practicamos examen clínico general y ginecológico en particular, biometría hemática, glicemia, uranálisis, citología funcional y Papanicolaou. A doce les tomamos biopsia de endometrio para estudio histológico.

A quienes alcanzaron a recibir cuatro inyecciones les practicamos a manera de control estos mismos exámenes más estudio histoquímico endometrial a cinco, y prueba de función tiroidea a seis, ochenta días después de la cuarta, de la quinta o de la sexta inyección. Todas las pacientes a quienes practicamos este control han continuado el método hasta la fecha, habiendo recibido cada una un total de siete a diez dosis.

Hemos entrevistado también a quienes por una $U$ otra razón abandonaron el método.

A ninguna de estas pacientes le suministramos terapia estrogénica ni derivados de la ergotamina para el control de sangrados anormales pues queríamos observar el real y vercladero efecto de la droga investigada.

III. Edad

CUADRO Nং 1

A. M. P. D. PARA CONTROL DE LA FERTILIDAD. - EDAD

\begin{tabular}{llcr}
\hline \multicolumn{1}{c}{ Edad } & Pacientes tratadas & $\%$ & Suspendieron la terapia \\
\hline 25 a 29 & 1 & 1,05 & 8 \\
30 a 34 & 12 & 12,61 & 20 \\
35 a 39 & 45 & 47,40 & 14 \\
40 a 45 & 37 & 38,94 & \\
Edad promedio: 38 años. & & & \\
\hline
\end{tabular}

Ocho pacientes del grupo comprendido entre los 30 y los 34 años suspendieron el método: 6 después de la primera inyección por amenorrea y dos después de la tercera por altera- ciones muy marcadas del ciclo menstrual.

La mayoría de las pacientes que aceptaron esta modalidad de trata- 
miento estuvieron por encima de los 35 años (82 pacientes), pero 34 de ellas lo abandonaron: 16 después de la primera inyección, once después de la segunda y siete después de la tercera.

El promedio de edad de las pacientes que aceptaron el método fue de 38 años.

\section{Efectos indeseables}

En siete pacientes encontramos un aumento de peso que fue de dos a ocho kilos $(7,3 \%)$.

En dos pacientes $(2,2 \%)$ observamos disminución de la líbido más depresión e inseguridad: abandonaron el método después de recibir la segunda y la tercera inyección respectivamente.

Constatamos otros efectos indeseables tales como cefálea, mastodinia, dolor pélvico, nerviosismo, cloasma, irritabilidad, náuseas, pero ni produjeron trauma síquico ni fueron factor determinante para abandonar el método; y vale la pena recordar que cuando se efectúan investigaciones de terapia ovulostática bajo cualquier modalidad, se encuentran efectos indeseables tanto en el grupo que se estudia como en el grupo que recibe placebo.

Pero el verdadero problema del A. M.P.D. estriba en las alteraciones del ciclo menstrual normal; encontramos una gran variedad de patrones que fueron desde patrones inaceptables (68 pacientes, $71,4 \%$ ), hasta amenorrea (en 12 pacientes, 12,6\%), con marcados cambios en el color y la viscosidad del sangrado, con toda la gama de molestias físicas, cambios de carácter y alteraciones síquicas.

Las alteraciones menstruales que observamos fueron: goteo o mancha- do que cesaba y se repetía de manera acíclica e imprevisible por períodos indeterminados en 33 pacientes $(34,7 \%)$; sangrado intermitente e irregular en intensidad y duración en 17 pacientes $(17,8 \%)$; marcado hipo $\checkmark$ opsomenorrea en 18 pacientes $(18,9 \%)$; y amenorrea en 12 pacientes $(12,6 \%)$. Sólamente en $13 \mathrm{pa}$ cientes $(13,8 \%)$ encontramos un ciclo menstrual más o menos similar al que tenían antes de la administración de la droga.

\section{CUADRO N: 2}

A. M. P. D. PARA CONTROL DE LA FERTILIDAD. EFECTOS INDESEABLES

\begin{tabular}{lcc}
\hline Efectos indeseables & $\begin{array}{c}\text { No } \\
\text { Pacientes }\end{array}$ & $\%$ \\
\hline Goteo o manchado persistente & 33 & 34,7 \\
Hipomenorrea u opsomenorrea & 18 & 18,9 \\
Sangrado intermitente & 17 & 17,8 \\
Amenorrea & 12 & 12,6 \\
Ansiedad o nerviosismo & 9 & 9,5 \\
Aumento de peso & 7 & 7,3 \\
Cloasma & 5 & 5,2 \\
Cefálea & 5 & 5,2 \\
Mastodinia & 4 & 4,2 \\
Irritabilidad & 4 & 4,2 \\
Mareos & 3 & 3,2 \\
Náuseas & 3 & 3,2 \\
Pérdida de la líbido & 2 & 2,2 \\
Edemas & 1 & 1,1 \\
Dolor pélvico & 1 & 1,1 \\
\hline
\end{tabular}

\section{Control posterior}

Cuarenta y dos pacientes abandonaron el método $(44,2 \%)$ : siete lo hicieron por amenorrea $(7,3 \%), 33$ por alteraciones muy marcadas de su patrón menstrual normal $(34,8 \%)$; y dos por pérdida de la líbido $(2,1 \%)$. Todas aquellas que lo abandonaron por amenorrea, menstruaron espontáneamente entre 8 y 14 meses después de la última dosis recibida. Y quienes desistieron por alteraciones menstruales diferentes a la amenorrea y que pudimos controlar demo- 
raron entre 6 y 10 meses para regularizar su ciclo en cantidad y duración. No estamos en capacidad de informar cuándo se reanuda la ovulación de manera espontánea después de la supresión de la droga, pero creemos que haya un intervalo prolongado e impronosticable.

A las 53 pacientes que no abandonaron el método les practicamos diversos controles clínicos y paraclínicos (biometría hemática, función hepática, pruebas de coagulación), ochenta días después de la cuarta, quinta o sexta inyección y no observamos alteraciones que pudiésemos atribuir al A.M.P.D. Practicamos también exámenes citológicos e histológicos.

A doce pacientes les hicimos curva de tolerancia a la glucosa después de haber recibido ocho inyecciones, sin que encontrásemos elevación alguna significativa $(5,14)$.

Los 53 frotis citológicos cervicales, (Papanicolaou) fueron negativos (grados $1 \circ 11$ ).

La citología funcional la tomamos por frotis vaginal en todas, y a diez pacientes les practicamos además urocitograma. Encontramos baja cariopignosis, células basales y parabasales y abundante agrupación.

A seis pacientes, cuatro en amenorrea y dos con ciclos totalmente irregulares les practicamos control de captación del T3 para función tiroidea y obtuvimos cifras compatibles con eutiroidismo.

Hicimos biopsia de endometrio a las cinco pacientes amenorreicas que continuaron con el método y en ellas encontramos escaso tejido endometrial con glándulas cilíndricas tapizadas por epitelio columnar bajo, es- troma de células fusiformes, muy poca actividad: endometrio atrófico.

La biopsia endometrial practicada a seis pacientes con sangrado intermitente o con manchado nos demostró escaso material con glándulas cilíndricas, epitelio parejo sin secreción, estroma de células pequeñias, fusiformes: endometrio débilmente proliferativo.

El estudio histoquímico endometrial practicado a cinco pacientes con problemas de sangrado indicó mínima cantidad de fosfatasa alcalina en el epitelio lo mismo que en la secreción glandular, glucógeno negativo, mucopolisacáridos ácidos negativos en epitelio glandular.

Cabe decir: ninguna biopsia nos mostró endometrio secretor, así fuese inicial o débilmente secretor.

Todas estas pacientes han continuado la terapia y han recibido un total de seis a nueve dosis cada una. En trece de ellas no se han presentado hasta la fecha alteraciones significativas en su ciclo menstrual. Treinta y cinco continúan con patrones inaceptables del ciclo, si bien observamos en la mayoría una tendencia hacia la opso-hipo-menorrea. $Y$ tres continúan en amenorrea desde la primera inyección (amenorrea de 15 a 24 meses).

Ninguna de las 95 pacientes ha quedado en embarazo durante el tiempo en que ha recibido esta terapia.

\section{Comentarios}

Aun cuando el número de pacientes observadas fue reducido, pudimos comprobar la efectividad del $A$. M.P.D. para el control de la natalidad en el $100 \%$ de los casos estudiados. 
Esta terapia ovulostática llama la atención a mujeres jóvenes; más cuando se les ilustra sobre las diferentes alteraciones del ciclo menstrual que puede ocasionar, la olvidan y dan preferencia a cualquier otra modalidad. Comportamiento similar pudimos observar en mujeres de mayor edad( aún en aquellas que frisan la menopausia, si bien en éstas el temor al embarazo puede ser tal que supere a las molestias que acarrea el método.

En general las pacientes de más de 35 años cuando están bien motivadas, aceptan con mayor facilidad las alteraciones menstruales, siendo mejor tolerada la amenorrea que los sangrados. Este problema de la amenorrea y de las alteraciones del ciclo menstrual ha sido descrito por la totalidad de los investigadores (8, 10, $16,15,18,23)$, quienes admiten que con la administración del A.M.P.D. el ciclo menstrual normal se elimina $y$ que en su reemplazo encontramos sangrado totalmente irregular e imprevisible.

Estas alteraciones tan marcadas y notorias del ciclo menstrual y estas amenorreas de tan larga duración nos autorizan a pensar que sólamente debemos usar el A.M.P.D. para control de la fertilidad en mujeres en finales de la tercera o de la cuarta década siempre y cuando hayan cumplido con su cuota deseada de familia. $Y$ por estas mismas razones opinamos que su empleo en nulíparas debe ser excepcionalmente raro, como en retardadas mentales, y aún debe ser proscrito, máxime cuando no podemos pronosticar con certeza cuál sea la respuesta de la función ovárica al uso prolongado de esta droga, ni menos cuál sea el futuro obstétrico de la mujer tratada.
Muy al contrario de lo que opinan algunos investigadores $(6,19)$ sostenemos que se debe motivar exhaustivamente a toda paciente a quien se vaya a administrar el A.M.P.D. con el fin de evitarle serias complicaciones que le alteren el equilibrio emocional. Recordemos que para toda mujer la aparición de la menstruación en fecha fija y con caracteres normales es un hecho básico, factor indiscutible de su equilibrio síquico.

Tampoco compartimos lo expresado por algunos autores en cuanto a la inmediata reversibilidad a ciclos normales cuando se suspende la droga $(16,17)$, ya que nuestra experiencia ha sido muy distinta. Pudimos sí comprobar la reversibilidad de la función ovárica en cinco pacientes: dos que habían abandonado el método por amenorrea y tres por serias alteraciones del ciclo, fueron embarazadas entre 13 y 17 meses después de la última dosis recibida y cursan en la actualidad gestaciones normales, muy bien controladas, que llevan de 12 a 15 semanas.

Todos los controles citológicos de cérvix que hemos practicado a las pacientes han sido negativos sin que hubiésemos encontrado los alarmantes resultados descritos por Powell en su reciente trabajo ( 8 ). Con todo, compartimos su opinión cual es la de practicar Papanicolaou a toda paciente que reciba contraceptivos inyectables $u$ orales ya que mientras no sea refutada no podemos pasar por alto la posibilidad de cáncer causado por los esteroides administrados bajo cualquier modalidad (1, 11 ).

Creemos con todo que el A.M.P.D. puede ser de gran utilidad en pacientes de mayor edad (tal vez superior a los 35 años), bien motivadas e instruídas cuando por uno $u$ otro moti- 
vo no puedan seguir otra modalidad para el control de la natalidad, como también para grupos de pacientes que vivan distantes de los centros de planificación.

\section{Resumen}

Se administran para el control de natalidad $150 \mathrm{mg}$. de Acetato de Medroxy Progesterona de Depósito por vía muscular cada 90 días a noventa y cinco pacientes, una nulípara de 25 años y 94 multíparas mayores de 30 años.

Cuarenta y dos abandonan el método entre la primera y la tercera inyección, y sólamente 53 continúan recibiendo la terapia.

Se analizan los efectos indeseables, haciendo énfasis en las marcadas alteraciones del ciclo menstrual, el que toma patrones inaceptables en el $84,3 \%$ de los casos.

Se estudian 53 pacientes ochenta días después de haberles aplicado la cuarta, quinta o sexta inyección, tanto desde el punto de vista clínico como citohormonal, histológico e histoquímico. dos.

Se comentan los resultados obteni-

Se hace énfasis en la seguridad del Acetato de Medroxy Progesterona de Depósito para el control de la natalidad.

\section{Summary}

$150 \mathrm{mg}$. of Medroxy Progesterone Acetate of Deposit (M.P.A.D.) are given by muscular means every 90 to 95 patients one nullipara aged 25 and 94 multiparae over 30 years of age.

Forty two abandoned the method between the first and the third injection and only 53 continued receiving the therapy.
Undesirable effects are analyzed with emphasis on the marked alterations of the menstruation cycle, which has un-acceptable patrons in $84,3 \%$ of the cases.

Fifth three patients are studied 80 days subsequent to the application of the fourth, fifth or sixth injection, both from the clinical aspect as of the cyto-hormonal histological histochemical aspects.

The results obtained are commented.

Emphasis is made on the safety of the M.P.A.D. for birth control.

\section{BIBLIOGRAFIA}

1 DE BRUX, J. et CHAMBE, G. Atypies cervicales au cours de traitements oestro-progestatifs. Comptes Rendus de la Soc. Fran. de Gynéc. 41: 123, 1971.

2 DUARTE-CONTRERAS, A., ZUÑIGA R. Progestógenos. Rev. Colomb. Obst. Gin. 14: 569, 1963.

3 DUARTE-CONTRERAS, A., ZUÑIGA, R. Y URIBE, G. Terapia secuencial. Rev. Colomb. Obst. Gin. 17: 255, 1966.

4 DUARTE-CONTRERAS, A., ZUÑIGA, R. y URIBE, G. Regulación de la fertilidad con terapia progestacional sin ovulostasis. Rev. Colomb. Obst. Gin. 19: 15, 1968.

5 GERSHBERG, H., ZORRILLA, E., HERNANDEZ, A. and HULSE, M. Effects of Medroxy Progesterone Acetate on serum insulin and growth hormone levels in diabetic and potentil diabetics. Obstet. Gynec. 33: 383. 1969.

6 HARNECKER, J. and al. Procedings, Sixth World Congress of Fertility and Sterility. Tel Aviv, May 20-27, 1968.

7 LARRAÑAGA, A. y KESSERU, E. Experiencia clínica con un anticonceptivo hormonal inyectable de depósito. Memor. V Congreso Lat. Amer. Obst. Gin. Viña del Mar. T. II: $713,1966$.

8 POWELL, Jr. L. C. and SEYMOUR, R. J. Effects of Depot Medroxy Progesterone acetate as a contraceptive agent. Am. J. Obstet. Gynec. 110: 36, 1971. 
9 PULIDO, M., RODRIGUEZ, R. y WILLIMITZER, H. Enantato de Nor etisterona como anticonceptivo de depósito. II Congreso Bolivariano Endocr. Lima, 1969.

10 RICE-WRAY, E., BECERRA, C. Y MAGUCO, $M$. Regulación de la fertilidad mediante una forma inyectable de una combinación progestógeno estrógeno. Proc. VI Congr. Pan. Am. Endocr. México, 1965.

11 RICE-WRAY, E. Y GOLDZIEHER, J. E. Efectos colaterales con esteroides anticonceptivos. Obstet. Ginec. Méx. 173: 253, 1971.

12 RODRIGUEZ, R. El capronato de 17-alfa-hidroxy-19-Nor-progesterona como agente anticonceptivo inyectable de depósito. Rev. Colomb. Obst. Gin. 20: 247, 1969.

13 RODRIGUEZ, R. Anticoncepción con dos gestógenos inyectables de depósito. Mem. Simposio Esteroides Sexuales. Bogotá, 265, 1968.

14 RODRIGUEZ-ARGUELLES, J. Anticonceptivos de depósito y metabolismo glúcido. Ginec. Obstet. Méx. 174: 353, 1971.

15 RUBIN, A. Prolonget amenorrhea following medroxyprogesterone. Am. J. Obstet. Gynec. 88: 1092, 1964.
16 RUBIO, B. y cols. Características del sangrado cuando se emplea medroxy progesterona, un progestgeno de depósito. Ginec. Obstet. Méx. 151: 503, 1969.

17 RUBIO, B. y cols. Proc. Sixth World Congress of Fertility and Sterility. Tel Aviv, May 20-27, 1968.

18 SEYMOUR, R. J. and POWELL, Jr. L. C. Depo Medroxy Progesterone acetate as a contraceptive. Obstet. Gynec. 36: 589, 1970.

19 TYLE, G. T. Sixth World Congress of Fertility and Sterility. Tel Aviv, May 20-27, 1968.

20 TYLER, E. Un estudio de anticonceptivos inyectables. Proc. VIII Conferencia Mundial I. P. P. F. Santiago Chile, 1967.

21 ZAÑARTU, J., RICE-WRAY, E. and GOLDZIEHER, J. W. Fertility control with longsetin inyectable steroids. Obstet. Gynec. 28: 513, 1966.

22 ZAÑARTU, J. A new approach to fertility control: longacting inyectable progesterone. Advances in Fertility Control. 3: 41, 1968.

23 ZARTMAN, E. R. Proc. Sixth World Congress of Fertility and Sterility. Tel Aviv, May 20-27, 1968. 